\title{
Psychological, endocrine, and neural correlates of attentional bias in subclinical depression
}

\author{
Katarina Dedovic ${ }^{\mathrm{a}, \mathrm{b}}$, Saskia Giebl ${ }^{\mathrm{c}}$, Annie Duchesne ${ }^{\mathrm{a}, \mathrm{d}}$, Sonja D. Lue ${ }^{\mathrm{a}}$, Julie Andrews ${ }^{\mathrm{a}}$, \\ Simona Efanov ${ }^{a}$, Veronika Engert ${ }^{a, e}$, Thomas Beaudry ${ }^{f}$, Mark W. Baldwin ${ }^{g}$ and Jens \\ C. Pruessner ${ }^{\mathrm{a}, \mathrm{f}}$
}

aDDouglas Institute Research Centre, McGill University, Boulevard Lasalle Verdun, QC, Canada; ${ }^{b}$ Social and Affective Neuroscience Laboratory, University of California, Los Angeles, CA, USA; 'Department of Psychology, University of California, Los Angeles, CA, USA; ${ }^{d}$ Department of Biology, Bishop's University, Sherbrooke, QC, Canada; ${ }^{\text {eDepartment }}$ of Social Neuroscience, Max Planck Institute for Human Cognitive and Brain Sciences, Leipzig, Germany; ${ }^{f}$ McGill Centre for studies in Aging, McGill University, Verdun, Québec, Canada; ${ }^{9}$ Department of Psychology, McGill University, QC, Canada

\begin{abstract}
Background: Our knowledge with respect to psychological, endocrine, and neural correlates of attentional bias in individuals with high vulnerability to developing depression - the subclinically depressed, still remains limited. Design: The study used a $2 \times 2$ mixed design. Methods: Attentional bias toward happy and sad faces in healthy $(N=26)$ and subclinically depressed individuals $(N=22)$ was assessed via a neuroimaging dot-probe attention task. Participants also completed trait and state psychological measures and provided saliva samples for cortisol analysis. Results: The subclinical group showed attentional bias toward happy faces; past use of problem-focused coping strategies when dealing with a personally relevant stressor as well as state levels of anxiety, together, contributed to this bias. In the control group, the happy attentional bias was positively correlated with activity in the right caudate. In the subclinical group, the bias was negatively associated with the left fusiform gyrus and positively with the left inferior parietal lobule and bilateral putamen. We observed group differences in association between cortisol levels during the task and neural activity during happy attentional bias processing within the key regions involved in attention. Conclusions: The attentional bias toward happy faces may reflect an active coping attempt by the subclinical participants.
\end{abstract}

Attention, memory, and attribution biases toward negative information contribute to depression onset and progression (Mathews \& MacLeod, 2005). Specifically, attentional bias toward negative information hinders emotion regulation and employment of positive coping strategies, particularly during periods of challenge or stress, and thus exacerbates the course of depression (reviewed in Gotlib \& Joormann, 2010). On the other hand, biased attention toward positive information has been shown to contribute to successful emotion regulation resulting in lower mood reactivity in healthy (e.g. Taylor, Bomyea, \& Amir, 2011) and even better treatment outcome in clinical populations (e.g. Browning, Holmes, Charles, Cowen, \& Harmer, 2012). Significant investigative effort has been made in furthering our understanding of the role of attention bias toward specific valenced 
stimuli and vulnerability and resilience to Major Depressive Disorder (MDD) (e.g. Foland-Ross \& Gotlib, 2012; Gotlib, Krasnoperova, Yue, \& Joormann, 2004; Peckham, McHugh, \& Otto, 2010). Nevertheless, despite this rich literature, some gaps in our knowledge still persist. For example, it is unclear how certain psychological and endocrine factors related to depression might be associated with attentional bias scores in healthy individuals and those with specific vulnerability to depression - the subclinically depressed. In addition, it is unknown how the neural mechanisms that underlie attentional bias may be disturbed in the subclinically depressed. As in attentional bias and anxiety research (Price et al., 2014), this line of investigation could provide clues for further mechanistic treatment refinement (e.g. neurofeedback or neurostimulation) and contribute to improvement of targeted prevention and intervention strategies. To address this gap in the field, we examined psychological (for example mood and coping), endocrine (cortisol) and neural correlates of attentional bias in healthy young adults that show varying levels of depressive tendencies, at a subclinical level.

Although different paradigms have been used to assess attentional bias (e.g. dot-probe task, (MacLeod, Mathews, \& Tata, 1986), emotional Stroop task (Mathews \& MacLeod, 1985; Mogg, Mathews, \& Weinman, 1989), and deployment of attention task (Gotlib, McLachian, \& Katz, 1988), results from a recent meta-analysis suggest that the dot-probe paradigm is most robust in detecting attentional bias (Peckham et al., 2010). In a dot-probe task, emotional and neutral stimuli are presented simultaneously; they are then removed and replaced by a target - usually a dot - appearing in the place of one of the stimuli, and participants are required to submit a response typically regarding the location of the target. Attentional bias is then determined by subtracting the reaction time related to detection of the target replacing the emotional stimulus (congruent condition) from the reaction time when detecting the probe appearing in the place of the neutral stimulus (incongruent condition). By presenting an emotional stimulus simultaneously with a neutral stimulus, the dotprobe task assesses relative attentional allocation more directly than the emotional Stroop task (Peckham et al., 2010). Notably, when the stimuli in the dot-probe task are presented for longer durations (1000 ms+), positive attentional bias score is typically interpreted to mean later stage allocation of attention to the valenced stimulus, while negative attentional bias score as allocation of attention away from the valenced stimulus (e.g. Shane \& Peterson, 2007). Importantly, with respect to attentional bias as related to depression, both the attentional bias toward negative information and attentional bias away from positive information have been observed; however, these have been found to be unrelated to each other, suggesting that both biases may play an important role in dysphoric information processing (Shane \& Peterson, 2007). Thus, in the current study we use both negative and positive stimuli coupled with neutral stimuli to be able to assess both bias types in the subclinically depressed adults.

We focused on the subclinical depression since it has been suggested that the subclinical depression may represent a milder condition on the depression severity continuum (Lewinsohn, Klein, Durbin, Seeley, \& Rohde, 2003; Rivas-Vazquez, Saffa-Biller, Ruiz, Blais, \& Rivas-Vazquez, 2004; Solomon, Haaga, \& Arnow, 2001) and that it may represent the precursor for the full disorder (Shankman et al., 2009). Here, subclinical depression (dysphoria) was assessed as scoring above a cut-off point for the normal range of depressive mood and below a cut-off point for the clinical range of depressive mood on a self-rating depression inventory that assesses symptom severity (Beck \& Steer, 1987). A recent study revealed that assessment of subclinical depression either via symptom counting method or assessment of symptom severity was associated with functional impairment in daily life; however, the symptom severity assessment was found to be more suitable to measure clinically relevant subclinical depression (Karsten, Hartman, Ormel, Nolen, \& Penninx, 2010).

Previous studies on subclinically depressed adults have shown a mood-congruent bias on the attention dot-probe task for both induced and naturally occurring dysphoria (Bradley, Mogg, \& Lee, 1997). Similarly, another study showed that, compared to non-dysphoric individuals, dysphoric participants allocated greater attention to depression-specific stimuli and less attention to positive stimuli (Shane \& Peterson, 2007). Similar findings have also been reported in a study on healthy girls at risk for depression due to their mother's psychopathology; these girls showed attentional 
bias toward negative facial expressions following a negative mood induction (Joormann, Talbot, \& Gotlib, 2007).

Although attentional bias toward positive information has been less frequently reported there is evidence that attentional bias toward positive information may be adaptive and can contribute to positive health outcomes. While some studies reported no attentional bias in healthy participants (for example Gotlib et al., 2004), others have put forward evidence that healthy controls show attentional bias toward happy faces (or they may avoid sad faces) (Joormann et al., 2007). Furthermore, healthy girls without family risk of depression showed attentional bias toward positive facial expressions following a negative mood induction (Joormann et al., 2007), effectively evoking attentional bias toward positive information as an emotional regulation strategy. Also, a study using attentional paradigm to train remitted depressed patients to pay attention to positive stimuli has shown that the training results in a decrease of residual depressive symptoms (Browning et al., 2012). This line of studies suggests that attentional bias toward positive information (or away from negative) may offer a degree of buffering regarding emotional reactivity.

Psychological factors such as trait and induced mood levels (depression or anxiety) (e.g. Bradley et al., 1997; Joormann et al., 2007), temperament (e.g. Helzer, Connor-Smith, \& Reed, 2009), and coping style (Klucken et al., 2010; Luecken, Tartaro, \& Appelhans, 2004) may influence one's attentional bias toward positive or negative information. In addition, biological factors such as levels of cortisol, an important marker of hypothalamic-pituitary-adrenal (HPA) axis function both in times of stress and rest, seem to be associated with attentional bias; however, the nature of this association remains unclear. For example, basal levels of cortisol were found to be negatively associated with bias to threatening words, and participants with less cortisol change in response to a psychological stress had greater increase in attentional bias scores for threatening stimuli (McHugh, Behar, Gutner, Geem, \& Otto, 2010); however, in an independent study by the same group the findings could not be replicated (McHugh et al., 2010). Others found that participants who showed a cortisol response to a mental arithmetic task had an increase in attentional bias toward depression-related stimulus following the task (Tsumura \& Shimada, 2012).

With respect to neural correlates of voluntary orientating and reorienting of attention, studies have implicated inferior parietal sulcus and temporal parietal junction respectively (e.g. Corbetta, Kincade, Ollinger, McAvoy, \& Shulman, 2000). A neuroimaging study examining specifically attentional bias toward sad words using a Stroop task in a clinically depressed sample, revealed that the engagement of rostral anterior cingulate cortex (ACC) and right precuneus during processing of sad words was greater in patients compared to controls (Mitterschiffthaler et al., 2008). Other studies examining processing of sad or negative information in depressed samples using several cognitive tasks have also implicated attention control regions such as rostral ACC, as well as engagement of executive (dorsolateral prefrontal cortex, dIPFC) and inhibitory (ventrolateral prefrontal cortex, VLPFC) regions (reviewed in Foland-Ross \& Gotlib, 2012). Other studies on the role of emotion in modulating attention focused on processing of threatening stimuli (e.g. fearful or angry faces) and revealed an important role for amygdala, extrastriate cortex, and parietal regions (reviewed in Vuilleumier, 2005).

Neuroimaging studies examining attentional bias toward happy faces typically reveal much greater effects regarding threatening stimuli compared to positive. Nevertheless, a study using an attentionshifting task revealed that while healthy participants deployed early attention to both sad and happy expressions (as assessed via P200 in the frontal region), dysphoric individuals only deployed early attention to sad (Buodo, Mento, Sarlo, \& Palomba, 2015). Another study revealed engagement of left caudate and left cuneus during attention bias toward happy faces (Lindstrom et al., 2009).

To date, it is still unknown what are the neural correlates of attention bias to sad and happy faces, in a subclinically depressed sample.

In the present study, we assessed attentional bias toward happy and sad faces presented for 1000 $\mathrm{ms}$ in healthy participants and those with heightened subclinical levels of depression. We hypothesized that the at-risk group would show a bias toward sad faces, and that this bias would be associated with a maladaptive psychological and endocrine profile (e.g. poor coping strategies, higher state 
levels of depression, and change in cortisol levels during the attentional bias task - given the equivocal results in the literature regarding cortisol levels and attentional bias, we did not hypothesize direction of this association). Also, we expected to observe group differences in neural correlates of sad and happy attentional bias within neural regions previously observed to be involved in attentional processing such as rostral ACC, caudate, inferior parietal regions, and amygdala and extrastriate cortex. Extrapolating from previous studies, we expected that, in the subclinically depressed group, attentional bias toward sad faces would be associated with heightened activity in these regions, particularly rostral ACC, inferior parietal regions, amygdala and extrastriate cortex. Finally, we also conducted exploratory analyses to examine possible group differences with respect to how cortisol levels during the attentional bias task may interact with neural correlates of attentional bias.

\section{Methods}

\section{Subjects}

Below, we outline how we determined our sample size, data exclusions, manipulations, and measures in the study. As a part of a larger neuroimaging study, we recruited 64 (30 men; 34 women) righthanded, healthy college students (mean age $=21.9 \pm 2.5$ ). Based on self-report measures (in-house socio-demographic and psychological questionnaires), it was determined that all subjects were non-smokers, and had no prior and/or present neurological or psychiatric illness, and were not taking any medication that could influence cortisol secretion. Furthermore, all subjects further met the safety requirements for participation in a functional Magnetic Resonance Imaging (fMRI) study. Also, they reported no current diagnosis or history of claustrophobia or Axis I disorders on the self-report questionnaires. The final selection was based on their score on the Beck Depression Inventory (BDI; Beck \& Steer, 1987). Based on the published BDI cut-off scores (Beck \& Steer, 1987), the subjects were initially recruited to either a control group $(B D I \leq 9 ; N=33)$ or a subclinically depressed group $(10 \leq \mathrm{BDI} \leq 18 ; N=31)$.

On the scan day, subjects completed the Hamilton Depression Inventory (HDI) (Reynolds, 1995), as well as the Montgomery-Asberg Depression Rating Scale Self-Assessment (MADRS-S) (Svanborg \& Asberg, 1994) as a crosscheck for BDI depression levels obtained at the time of recruitment. We observed that several subjects had scored at clinical depression levels either on the HDI or MADRS-S at that time. These subjects represented high-risk subclinical subjects and were advised to seek professional counsel and were given a referral letter. We did not include these participants for the subsequent analyses. Instead, we opted to focus only on the healthy participants and those participants whose levels of depression remained at subclinical levels throughout the duration of the study (27.6 days \pm 11.4 passed from the recruitment in the study until completion of the main testing session).

Finally, inspection of the data revealed that seven subjects had to be excluded for the following reasons: one participant was falling asleep during the task; two participants did not have any correct trials; for one participant, task log files were not saved; for two participants, the task response button box malfunctioned; for one participant, neuroimaging data were lost from the scanner console. Therefore, the final number of participants was 26 healthy (CTRL; 12 men; 14 women) and 22 subclinical (SUB; 12 men; 10 women).

The Institutional Review Board (IRB) of McGill University approved the study, and informed consent was obtained prior to participation in accordance with the requirements of the McGill IRB from all subjects.

\section{Procedure}

On average, two weeks prior to the testing day, subjects came to Montreal Neurological Institute and completed a face-rating task. Faces used were selected from a database of standardized facial images (Lundqvist, Flykt, \& Ohman, 1998). All images were modified using Adobe Photoshop 7.0 (Adobe 
Systems, San Jose, CA) to achieve consistency in size and contrast, and were converted to grayscale. The task involved rating 15 male (10 which participants would see again in the fMRI scan and 5 filler faces) and 15 female faces (again, 10 which participants would see again in the fMRI scan and 5 filler faces) on valence from negative to positive; each face was presented once showing neutral, once showing sad, and once showing happy facial expression. Presentation of faces and expressions was randomized.

On the testing day, participants arrived at the Montreal Neurological Institute in the afternoon, 1 hour prior to the magnetic resonance imaging (MRI) scanning. The subjects were given several psychological questionnaires to complete. Fifteen minutes prior to entering the scanning room, a research assistant explained the procedure and tasks that would be performed in the fMRI scanner. Subjects were then introduced to the study investigator and exposed to three functional runs of an attentional bias dot-probe task that had been modified for neuroimaging environment, followed by a structural scan, and finally a challenging mental arithmetic task (Dedovic et al., 2005). Neural, physiological, and psychological data related to the mental arithmetic task have been published elsewhere (Dedovic et al., 2014) and therefore, are not treated here.

All participants had normal or corrected-to-normal vision during the examinations.

\section{Dot-probe task}

The attentional bias task employed in this study is based on the classical dot-probe task (Bradley, Mogg, Falla, \& Hamilton, 1998) and was adapted for neuroimaging environment. Participants completed the task over the course of three runs, each run lasting about 11 minutes. The first run was used as a practice run in order for subjects to get accustomed to being in the scanner and to get used to the task. The attentional bias scores were calculated from the typical emotional cue + target trials. Participants saw a cross hair for $500 \mathrm{~ms}$, followed by a pair of faces (the cue; one face with neutral expression and the other with emotional expression) for $1000 \mathrm{~ms}$. Once the picturepair had disappeared, a blank jitter card that lasted between 0 and 300 ms was presented, followed by a pair of dots (the target; either in vertical (:) or horizontal (.) orientation). The target appeared on the screen either on the side of the screen where the neutral face had been on, or on the side of the emotional face. Subjects were instructed to indicate what dot type is on the screen, as fast as possible. After submitting a response or after 2 seconds had passed (whichever came first), a cross hair appeared on the screen again and remained there for 3-5 seconds (long jitter). In total, subjects saw 40 cue + target happy trials and 40 cue + target sad trials; these trials were balanced for the side of the emotional picture, male and female faces, the type of dot target, and for congruency. Participants also completed 16 cue only trials for each emotion (consisted of seeing the cross-card, then the pairs of faces, followed by the long jitter), 28 empty trials (cross card lasting $500 \mathrm{~ms}+$ the long jitter); however, both behavioral and neuroimaging analyses focus on emotional cue + target trials specifically. We also included neutral-neutral trials (32 Cue + target; 24 Cue only). All trial types were pseudorandomized in order, so that their occurrence was similarly distributed across the run.

To calculate attentional bias scores and minimize influence of outliers, we followed a standard procedure (Gotlib et al., 2004): firstly, reaction times (RT) for only correct trials were used in determination of the attentional bias; secondly, trials with RT less than $100 \mathrm{~ms}$ or greater than $1000 \mathrm{~ms}$ were excluded as they are likely to reflect anticipation errors and lapses in concentration, respectively. The attentional bias to a given emotional facial expression was determined by calculating reaction time during non-congruent trials (the condition where the dot target appears behind the neutral face) minus congruent trials (the condition where the dot target appeared where the emotional face had been) (equation from Karin Mogg, Bradley, \& Williams, 1995): attentional bias score $=1 / 2$ * $[($ RpLe - RpRe $)+($ LpRe - LpLe) $]$, where R stands for right position, $L$ for left position, and $p$ for probe and e for emotional face; this is equivalent to calculating Mean RT incongruent - Mean RT congruent score. A positive score indicates that participant is attending to the location of the emotional face relative to the matched neutral face, while a negative score indicates diverting of attention from the spatial location of the emotional face compared to the location of the neutral face. 


\section{Psychological assessment}

In addition to the MADRS and HDI, we also administered additional questionnaires that assessed participants' psychological states and traits, including the Ways of coping questionnaire (WAYS) to assess participants coping strategies when faced with a stressor (Folkman \& Lazarous, 1985). Specifically, participants were asked to identify a stressful situation that they had experienced in the last month and then indicate to what extent they engaged in various coping strategies to deal with this stressful event (for example "I made a plan and followed it", "I criticized and lectured myself"). The WAYS assesses five types of coping strategies: problem focused, seeking social support, blamed self, wishful thinking, and avoidance. Participants' state levels of anxiety and depression were also assessed via the Profile of Mood States (McNair, Lorr, \& Droppleman, 1992) prior to the scan.

\section{Saliva sampling}

Using the salivette sampling device (Sarstedt Inc, Quebec City, Quebec, Canada), we collected two saliva samples related to the attentional bias task: the first saliva sample was taken before the scan (the timing varied between 12:00 pm and 5:17 pm), when subjects were seated at the scanning bench, and the second saliva sample was taken after the second attentional bias task run, approximately 30 minutes following the first sample. The saliva samples were stored in the laboratory freezer at $-20^{\circ} \mathrm{C}$ until analysis. Samples were analyzed via a time-resolved fluorescence immunoassay, of which intra- and inter-assay variability have been shown to be less than $10 \%$ and $12 \%$, respectively (Dressendorfer, Kirschbaum, Rohde, Stahl, \& Strasburger, 1992).

\section{Behavioral statistical analysis}

Group differences on psychological variables were assessed using univariate analysis of variance (ANOVA) with study group and sex as between factors. Differences with respect to attentional bias used mixed design ANOVA with run and attentional bias valence as repeated measures and group and gender as between factors. Differences with respect to change in cortisol were also assessed via a mixed-design ANOVA with cortisol levels across the two points as repeated measures, and group and sex as between factors. Since the cortisol values were not normally distributed, we log transformed the data for the statistical analyses. Factors that are known to influence these values were included as a covariate (See Result section for specific details).

Whenever the assumption of sphericity was violated, we applied Greenhouse-Geisser correction. Significant interactions were followed up with corresponding $t$-tests. All analyses were conducted with SPSS Statistics software v21. Effects were deemed to be significant when at $p \leq .05$.

Effect sizes in form of Partial $\eta^{2}$ for ANOVA analyses were calculated via SPSS; effect sizes in form of Cohen's $d$ for $t$-tests were calculated using powerAnalysis package developed by Felix Yanhui Fan (https://github.com/cran/powerAnalysis) for statistical program R. When appropriate, we also provide 95\% Confidence Intervals (CI) for the results, which were calculated using the online Confidence Interval calculator for the population mean (http://www.danielsoper.com/statcalc3/calc.aspx?id=96) developed by Daniel Soper, PhD.; the $95 \% \mathrm{Cl}$ for Cohen's $d$ statistic for one-sample and twosample $t$-test was calculated using SPSS via syntax developed by Karl Wuensch, PhD. (http://core. ecu.edu/psyc/wuenschk/SPSS/Cl-d-SPSS.pdf), while for the paired $t$-tests MBESS library for $\mathrm{R}$ was used (http://www3.nd.edu/ kkelley/site/MBESS.html).

\section{Neuroimaging analysis and processing}

The subjects were scanned in a 1.5 T Siemens scanner. For the structural images, standard 3D gradient-echo pulse sequence was used, with the field of view of $256 \mathrm{~mm}$, the voxel size of $1 \times 1 \times 1 \mathrm{~mm}$, TR of $22 \mathrm{~ms}$, TE of $9.2 \mathrm{~ms}$, and a flip angle of $30^{\circ}$.

During each functional run, 276 whole-brain BOLD Mosaic $64 \mathrm{~T} 2^{*}$-weighted echo-planar images were acquired transversely, along the direction of the anterior commissure to the posterior 
commissure line minus $30^{\circ}$ (voxel size $=4 \times 4 \times 5 \mathrm{~mm}$; slice number $=28$; order of slice acquisition $=$ interleaved; $\mathrm{TR}=2370 \mathrm{~ms} ; \mathrm{TE}=50 \mathrm{~ms}$; flip angle $=90^{\circ}$, matrix $=64 \times 64$, field of view $=256 \mathrm{~mm}$ ).

We preprocessed the structural and functional data with FMRIB Software Library (FSL) tools (Smith et al., 2004; Woolrich et al., 2009) as well as tools contained within the Statistical Parametric Mapping software package (SPM8) (http://www.fil.ion.ucl.ac.uk/spm/).

First, the Brain Extraction Tool (BET) was used to remove any non-brain tissue from both structural and functional images (Smith et al., 2004). Following this procedure, images were manually inspected to verify that the procedure did not affect brain tissue. The functional raw data were motion corrected by FMRIB's Linear Image Registration tool (mcflirt; http://www.fmrib.ox.ac.uk/fsl/mcflirt/index.html) which conducts linear inter-modal registration with 6 degrees of freedom, aligning each functional frame to the middle frame in each run (Jenkinson, Bannister, Brady, \& Smith, 2002).

Furthermore, the structural and functional images were registered to the MNI template image (MNI152_T1_2mm_brain.nii.gz) using a three-step procedure and the FMRIB's Linear Image Registration tool (flirt; http://www.fmrib.ox.ac.uk/analysis/research/flirt/) (Jenkinson et al., 2002).

Finally, the normalized images were then transferred into SPM8 and smoothed using a $6 \mathrm{~mm}$ fullwidth-half-maximum Gaussian kernel in order to spatially smooth the data and reduce noise.

Modeling and analysis of data was conducted using SPM8. Only the correct cue + target trials were considered for calculations of contrasts of interest. Each event was modeled starting at the appearance of the cue card and ending with the submission of the correct response. To account for the presence of other trial types, in addition to cue + target trials, we also modeled cue only trials, as well as the long jitter that followed submission of the response to cue + target trials; we also included a junk regressor of no interest that included all incorrect trials. In addition, to control for possible contribution of movement to the changes in brain activity, we also included the movement parameters as regressors of no interest. Empty trials and other cross cards were not modeled. The main contrast of interest at the first level was similar as in the behavioral data analyses: namely, incongruent > congruent trials for each emotional facial expression.

High-pass temporal filtering of the data and the model was set to $160 \mathrm{~s}$. To account for serial correlations in fMRI time series due to aliased biorhythms and unmodelled neuronal activity, we used an autoregressive AR (1) model during Classical (ReML) parameter estimation.

At the second level, we aimed to investigate group differences with respect to neural regions that are associated with the behavioral attentional bias. To this end, the contrast images for incongruent $>$ congruent contrast were used in a multiple regression model. A multiple regression model included the following regressors: the first one coded for the CTRL group, the second one for the SUB group, third for the attentional bias scores for CTRL group, with the last regressor containing attentional bias scores for the SUB group. Sex was also included as a covariate of no interest. The contrast of interest was CTRL > SUB and CTRL < SUB for neural regions associated with attentional bias score, thus the contrast vectors were $000001-1$ (CTRL > SUB), and $00000-1$ 1(CTRL 0 <SUB) for the whole brain correlational analyses.

For all neuroimaging analyses, in order to balance both Type I and Type II error rates, we employed the recommended combination of intensity and cluster size thresholds of $p<.005,20$ voxels for establishing significance (Lieberman \& Cunningham, 2009).

Significant findings are displayed using the software xjView (http://www.alivelearn.net/xjview), on the BET-processed MNI 152 template.

\section{Results}

\section{Demographics}

As expected, the SUB group had higher levels of depression compared to the CTRL group as assessed via BDI $\left(F(1,44)=144.8, p<.001\right.$, Partial $\left.\eta^{2}=.77\right)$, HDI $\left(F(1,43)=26.9, p<.001\right.$, Partial $\left.\eta^{2}=.39\right)$ and MADRS $\left(F(1,44)=12.4, p=.001\right.$, Partial $\left.\eta^{2}=.22\right)$. The SUB group also had higher state measures of 
depression $\left(F(1,44)=10.1, p=.003\right.$, Partial $\left.\eta^{2}=.19\right)$, and exhibited tendency for heightened anxiety levels $\left(F(1,44)=2.52, p=.12\right.$, Partial $\left.\eta^{2}=.05\right)$. The groups did not differ with respect to coping strategies employed on any of the WAYS of coping subscales. For the results regarding gender and gender $\times$ group interaction please see $\mathrm{SI}$.

\section{Attentional bias: only the subclinical group shows a positive bias toward happy faces}

All participants were able to distinguish sad, neutral, and happy facial expressions (see SI). Groups did not differ with respect to mean RT for each trial type or mean number of incorrect trials (Table 1).

The ANOVA examining effect of group, gender, run number, and valence on attentional bias scores yielded a significant valence $\times$ group interaction $\left(F(1,44)=4.27, p=.045\right.$, Partial $\left.\eta^{2}=.09\right)$ (Figure 1; Raw RT for each condition can be found in Table 1). Specifically, participants in the SUB group showed a significant difference between attentional bias for happy faces (Mean \pm Standard Deviation, $18.99 \pm 27.87,95 \% \mathrm{Cl}: 6.63 \leq x \leq 31.35)$ compared to sad faces $(-4.51 \pm 30.51,95 \% \mathrm{Cl}$ : $-18.04 \leq x \leq 9.02)(t(21)=2.53, p=.02$, Cohen's $d=0.55,95 \% \mathrm{Cl}: 0.085 \leq d \leq 0.98)$. To test whether the observed differences are due to attentional bias toward happy faces or sad faces, we conducted a simple $t$-test to assess which attentional bias is different from zero. The analysis showed that the attentional bias for happy faces in the SUB group was greater than zero $(t(21)=3.20, p=.045$, Cohen's $d=0.70,95 \% \mathrm{Cl}: .21 \leq d \leq 1.14)$ indicating that the subclinical participants were attending to the happy faces.

Furthermore, the SUB group showed a greater attentional bias for happy faces (Mean \pm Standard Deviation, $18.99 \pm 27.87,95 \% \mathrm{Cl}: 6.63 \leq x \leq 31.35)$ compared to the control group $(-3.85 \pm 40.98,95 \%$ Cl: $-20.40 \leq x \leq 12.70)((t(44.1)=2.29, p=.027$, Cohen's $d=.66,95 \% \mathrm{Cl}: .076 \leq d \leq 1.24)$.

In order to further probe whether this attentional bias toward happy faces is related to orienting component or disengaging component of attentional bias, we calculated orienting index and disengaging index (Salemink, van den Hout, \& Kindt, 2007). The orienting index is calculated by subtracting mean RT for dots replacing happy faces in the presence of neutral faces (congruent trials) from mean RT on neutral-neutral cue + target trials - a positive score indicates faster response to targets appearing after the happy faces compared to neutral. The disengagement index is obtained by subtracting the mean RT for neutral-neutral cue + target trials from the mean RT for the happy faces incongruent trials - a positive score here indicates a slower response to neutral face in the presence of an emotional face compared to when paired with neutral face (Salemink et al., 2007). We conducted a one-tailed $t$-test in the subclinical group to examine whether the orienting index or the disengagement index for happy faces is greater than zero. The results indicated that the mean orienting index for happy faces was greater than zero ((Mean \pm Standard Deviation, $14.73 \pm 37.87,95 \% \mathrm{Cl}:-2.06 \leq$ $x \leq 31.52), t(21)=1.83, p=.04$, Cohen's $d=0.40,95 \% \mathrm{Cl}:-.048 \leq d \leq .82)$ but disengagement index

Table 1. Mean RT and number of incorrect trials per each trial type and group.

\begin{tabular}{lcccc}
\hline & $\begin{array}{c}\text { Control } \\
\text { Reaction times (ms) } \\
\text { Mean } \pm \text { SD }\end{array}$ & $\begin{array}{c}\text { Subclinical } \\
\text { Reaction times (ms) } \\
\text { Mean } \pm \text { SD }\end{array}$ & $\begin{array}{c}\text { Control } \\
\text { Number of incorrect trials } \\
\text { Mean } \pm \text { SD }\end{array}$ & $\begin{array}{c}\text { Subclinical } \\
\text { Number of } \\
\text { incorrect trials } \\
\text { Mean } \pm \text { SD }\end{array}$ \\
\hline Happy-Neutral congruent & $665.7 \pm 113.2$ & $644.3 \pm 110.3$ & $0.02 \pm 0.1$ & $.04 \pm 0.14$ \\
Happy-Neutral incongruent & $666.5 \pm 111.9$ & $660.7 \pm 105.1$ & $.02 \pm 0.1$ & $.05 \pm .15$ \\
Neutral-Happy congruent & $669.3 \pm 117.9$ & $645.6 \pm 112.4$ & $.02 \pm 0.1$ & $.05 \pm .15$ \\
Neutral-Happy incongruent & $662.7 \pm 123.5$ & $665.8 \pm 107.9$ & $.04 \pm 0.14$ & $0.2 \pm .11$ \\
Sad-Neutral congruent & $656.5 \pm 117.6$ & $649.5 \pm 106.2$ & $.02 \pm 0.1$ & 0 \\
Sad-Neutral Incongruent & $671.5 \pm 114.7$ & $636.4 \pm 98.5$ & 0 & $.05 \pm .15$ \\
Neutral-Sad congruent & $667.2 \pm 109.2$ & $653.5 \pm 114.9$ & $.06 \pm 0.16$ & $.02 \pm .11$ \\
Neutral-Sad incongruent & $657.1 \pm 116.1$ & $657.6 \pm 106.8$ & $.04 \pm 0.14$ & $.05 \pm .15$ \\
Neutral-Neutral left & $668.6 \pm 105.7$ & $660.9 \pm 111.3$ & $.08 \pm 0.18$ & $.05 \pm .15$ \\
Neutral-Neutral right & $663.4 \pm 104.6$ & $658.5 \pm 106.0$ & $.10 \pm .20$ \\
\hline
\end{tabular}

Note: There were no significant group differences observed. SD, Standard deviation. 


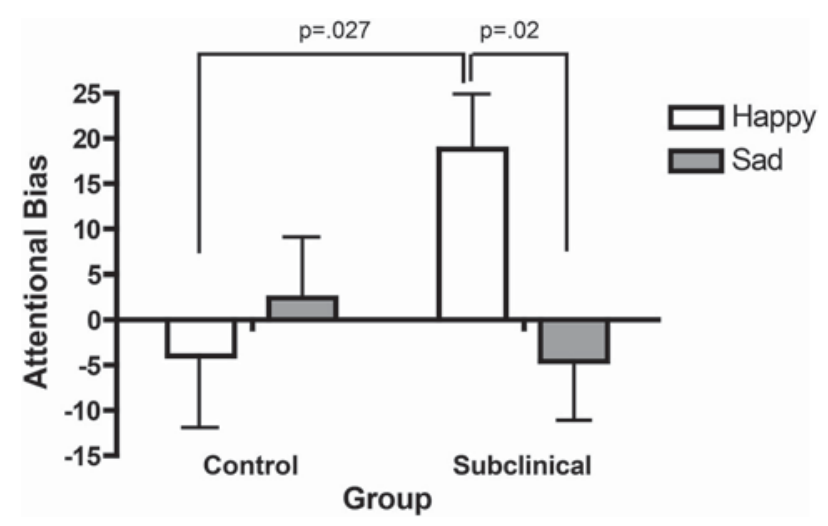

Figure 1. Group differences in attentional bias toward happy and sad faces. Participants in the subclinical group showed greater bias toward happy faces than sad faces, and showed a greater attentional bias for happy faces compared to controls. Mean \pm Standard Error.

was not $(3.58 \pm 27.78,95 \% \mathrm{Cl}:-8.74 \leq x \leq 15.90)(t(21)=.60, p=.276$, Cohen's $d=0.13,95 \% \mathrm{Cl}:-.29 \leq$ $d \leq .55)$.

\section{No change in cortisol levels observed over the course of the attentional bias task}

We assessed group differences in change over time in cortisol levels during the attentional bias task, while controlling for the time of day. This group $\times$ gender $\times$ time analysis of covariance did not reveal any significant effects or trends (CTRL group, Mean \pm Standard Deviation, $t 1=3.51 \pm 2.31 \mathrm{nmol} / \mathrm{L}$, $95 \% \mathrm{Cl}: 2.58 \leq x \leq 4.44, t 2=3.42 \pm 2.05 \mathrm{nmol} / \mathrm{L}, 95 \% \mathrm{Cl}: 2.59 \leq x \leq 4.25 ;$ SUB group, $t 1=2.53 \pm 1.13$ $\mathrm{nmol} / \mathrm{L}, 95 \% \mathrm{Cl}: 2.03 \leq x \leq 3.03, t 2=3.11 \pm 2.10 \mathrm{nmol} / \mathrm{L}, 95 \% \mathrm{Cl}: 2.18 \leq x \leq 4.04)$.

\section{State anxiety and problem-focused coping associated with attentional bias toward happy faces in the subclinical depression group}

To examine possible association of trait and state factors with attentional bias for positive information in the subclinical group, we conducted an exploratory hierarchical backward regression. We included the following factors: levels of problem focused, seeking social support, blamed self, wishful thinking, and avoidance coping strategies used to deal with a personally stressful situation in the month prior to the testing session, cortisol levels during the attentional bias (averaged levels of cortisol at time 1 and time 2), and state anxiety and depression levels prior to going into the scan, as independent variables. The final model was significant $\left(F(21)=3.65, p=.045\right.$, adjusted $\left.r^{2}=.20\right)$ and included a positive contribution of levels of problem-focused coping at a trend level $(t=2.04, p=.056)$ and a positive association with state anxiety also at a trend level $(t=2.08, p=.051)$ (Table 2$)$.

\section{Association between happy attentional bias and neural activity in caudate, left fusiform gyrus, left inferior parietal lobule, and bilateral putamen differ between the two groups}

Given that the behavioral data revealed group differences in the attentional bias to happy faces, we investigated group differences in neural correlates of attentional bias only for the happy faces. The multiple regression analyses revealed a CTRL > SUB effect with respect to association between neural activity and the attentional bias toward happy faces within the right caudate, and the left fusiform gyrus (Table 3). To decompose this effect, we extracted parameter estimates from these regions for incongruent $>$ congruent contrast and correlated these values with the attentional bias scores. This step revealed that in the CTRL group, the activity in the right caudate is positively associated 
Table 2. Linear model of predictors of bias toward happy faces in the subclinical group.

\begin{tabular}{lccrr}
\hline & $b$ & SE B & $\beta$ & $p$ \\
\hline Step 1 & & & & \\
Constant & -37.67 & 42.33 & .54 & .390 \\
Problem-focused coping & 21.08 & 9.64 & .12 & .048 \\
Seeking social support & 4.99 & 9.52 & -.35 & .609 \\
Blamed self & -11.96 & 8.16 & .39 & .167 \\
Wishful thinking & 18.11 & 15.85 & -.30 & .274 \\
Avoidance & -16.34 & 16.45 & .47 \\
Anxiety pre-task & 19.69 & 10.28 & .11 \\
Depression pre-task & 8.64 & 20.5 &. .20 & .078 \\
Average_cortisol & -.3 .76 & 4.78 & .680 \\
Step 7 & & & .446 \\
Constant & -35.27 & 22.39 & .41 & .111 \\
Problem-focused coping & 17.08 & 8.21 & .40 \\
Anxiety pre-task & 15.84 & 7.77 & .051 \\
\hline
\end{tabular}

Note: Adjusted $R^{2}$ for Step $7=.20$.

with the attentional bias scores; no association was found in the SUB group (Figure 2(a)). Conversely, in the SUB group only, activity in the left fusiform gyrus was negatively associated with increased attentional bias toward happy faces (Figure 2(b)).

It is important to note that extracted parameter estimates from these regions are taken from non-independent voxels, which can lead to biases in additional statistical analyses. Thus, the extracted parameter estimates are used simply to illustrate which association in which group is driving the observed effect at the whole brain level (CTRL $>S U B$, in this case); associated $r$ values are used to illustrate the direction of any significant correlation effect as contained within the primary analysis.

The multiple regression analyses also revealed a significant SUB > CTRL effect in the left inferior parietal lobule and the bilateral putamen (Table 3). Decomposing this effect revealed that, only in the SUB group, association between activity in these regions and the attentional bias toward happy faces was positive (Figures 2(c,d)).

\section{Association between average cortisol levels during the attentional bias task and neural activity}

Exploratory examination of the group differences with respect to correlation between average levels of cortisol during the attentional bias task and neural activity for the incongruent $>$ congruent contrast revealed a significant CTRL > SUB effect in the right postcentral gyrus (Table 4, Figure 3). Decomposing this effect revealed a negative association within the subclinical group specifically. In addition, the analysis revealed a significant SUB $>$ CTRL effect in the right anterior insula, right inferior parietal lobe, and right VLPFC. Decomposing this effect revealed a negative association between neural activity in the right anterior insula and right inferior parietal lobe and average cortisol levels

Table 3. Whole brain analyses reveal group differences in gray matter in association between happy attentional bias and neural activity in incongruent > congruent contrast for happy cue + target trials.

\begin{tabular}{|c|c|c|c|c|c|c|}
\hline & Anatomical region & $x$ & $y$ & $z$ & $t$ & $k$ \\
\hline Control> & Left fusiform & -36 & -48 & -6 & 3.31 & 28 \\
\hline \multirow[t]{2}{*}{ Subclinical } & Right caudate & 24 & 16 & 20 & 2.99 & 31 \\
\hline & & 18 & 22 & 18 & 2.69 & \\
\hline Subclinical> & Left inferior parietal lobe & -52 & -44 & 48 & 3.32 & 20 \\
\hline \multirow[t]{3}{*}{ Control } & Left inferior parietal lobe & -46 & -60 & 48 & 3.39 & 35 \\
\hline & Left putamen & -30 & -2 & 6 & 3.24 & 32 \\
\hline & Right putamen & 30 & -4 & 2 & 3.29 & 27 \\
\hline
\end{tabular}

Note: Montreal Neurological Institute coordinates; coordinate in italics connote peaks within a same cluster. 


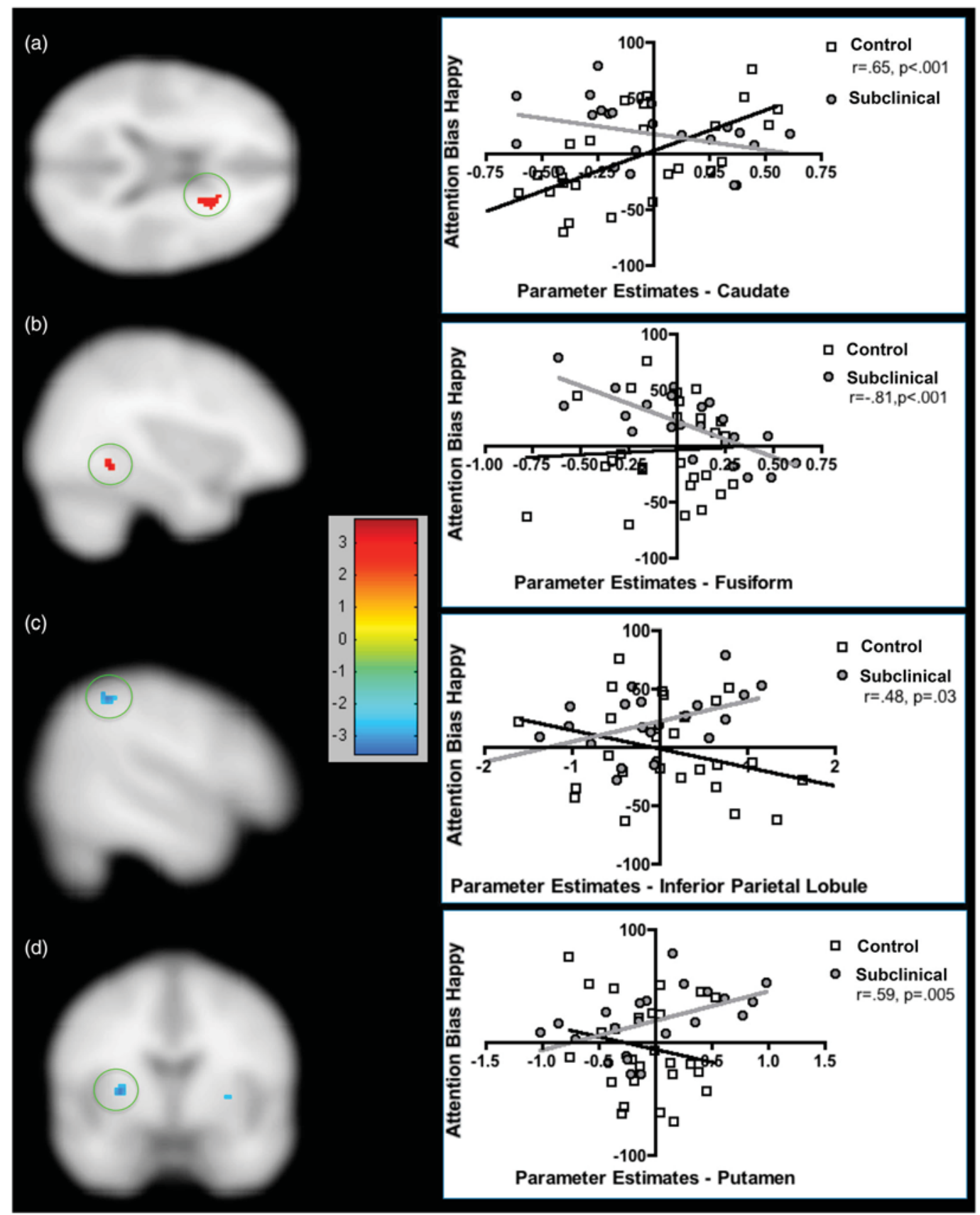

Figure 2. Group differences in gray matter in association between happy attentional bias and neural activity in incongruent $>$ congruent contrast for happy cue + target trials. Multiple regression analyses revealed a significant Control $>$ Subclinical effect with respect to association between neural activity and the attentional bias toward happy faces within the right caudate (Figure 2 (a)), and the left fusiform gyrus (Figure 2(b)). Also, the analyses revealed a significant Subclinical > Control effect in the left inferior parietal lobule (Figure 2(c)) and the left (Figure 2(d)) and right putamen. The graphs simply illustrate the decomposed effect; $r$ values are used to illustrate the direction of any significant correlation effect as contained within the primary analysis. All clusters are thresholded at intensity threshold of $p<.005$, extent threshold of 20 voxels. Areas in red connote the Control $>$ Subclinical contrast, while those in blue, the Subclinical > Control contrast. 
Table 4. Whole brain analyses reveal group differences in gray matter in association between average cortisol levels during the attentional bias task and neural activity in incongruent > congruent contrast for happy cue + target trials.

\begin{tabular}{|c|c|c|c|c|c|c|}
\hline & Anatomical region & $x$ & $y$ & $z$ & $t$ & $k$ \\
\hline $\begin{array}{l}\text { Control> } \\
\text { Subclinical }\end{array}$ & Right postcentral gyrus & 30 & -22 & 46 & 3.06 & 23 \\
\hline Subclinical> & Right anterior insula & 34 & 10 & -20 & 3.75 & 41 \\
\hline \multirow[t]{3}{*}{ Control } & & 28 & 16 & -16 & 2.86 & \\
\hline & Right inferior parietal lobe & 50 & -60 & 30 & 2.84 & 23 \\
\hline & Right VLPFC & 40 & 42 & -6 & 3.27 & 23 \\
\hline
\end{tabular}

Note: Montreal Neurological Institute coordinates; coordinate in italics connote peaks within a same cluster; VLPFC, ventrolateral prefrontal cortex.

among the control group; there was also a negative association between the VLPFC and cortisol in the control group but a positive one in the subclinically depressed group (Table 4, Figure 3).

\section{Discussion}

The present study investigated psychological, endocrine, and neural correlates of attentional biases in a sample of healthy university students with varying degrees of depression vulnerability: healthy controls and subclinically depressed. Contrary to our hypotheses, first, we observed that only participants in the subclinically depressed group attended to happy faces. Second, we observed that the degree to which subclinical participants endorsed using problem-focused strategies when coping with a particularly stressful event in the month prior to the testing session and greater levels of anxiety just prior to the testing session together significantly contributed to this bias. Moreover, we observed association between happy attentional bias and neural activity in key regions involved in voluntary sustained attention as well as reward and motivation processing, namely the left inferior parietal lobule and left fusiform gyrus, and basal ganglia. Furthermore, although no association was found between attentional bias toward happy faces and cortisol levels during the attentional bias task in the subclinically depressed group, we did observe group differences in association between cortisol levels and neural activity during happy attentional bias processing. Specifically, while controls showed an adaptive negative association within the key regions involved in attention - the right anterior insula, right inferior parietal lobe, and the right VLPFC, the subclinical group showed a maladaptive positive response with right postcentral gyrus and the right VLPFC. Overall, these results seem to suggest that subclinically depressed subjects who are able to engage active coping strategies and who have heightened levels of state anxiety may show some traces of resilience in form of adaptive or compensatory characteristics such the attentional bias toward positive information. However, notably, these individuals also inevitably show traces of vulnerability. Namely, the greater neural network that subserves the attentional bias toward happy faces seems to be modulated by levels of cortisol in a way that suggests its vulnerability to heightened levels of cortisol in the subclinical group. Therefore, overall, the findings reveal that some subclinically depressed participants may show degree of resilience by paying attention to positive information; however, this trace of resilience could be disrupted in contexts of higher levels of cortisol.

We posit that the bias toward happy faces reflects an active attempt by the subclinical participants to cope with their depressive tendencies. This proposition is in line with a study on healthy, depressed, and partially remitted patients that showed that reduced dysfunctional thinking (for example "If I fail to reach an important goal I am useless") in those with partial remission is primarily associated with increased effortful control of dysfunctional cognitive patterns, rather than reduced access to dysfunctional schemas (Sheppard \& Teasdale, 2004). Given that the subclinical participants tested in the present study were highly functional university students, it may be possible that they have introspective knowledge of their vulnerability, and are engaging in emotional regulation, including exhibiting an attentional bias toward positive information. Indeed, it has been suggested 

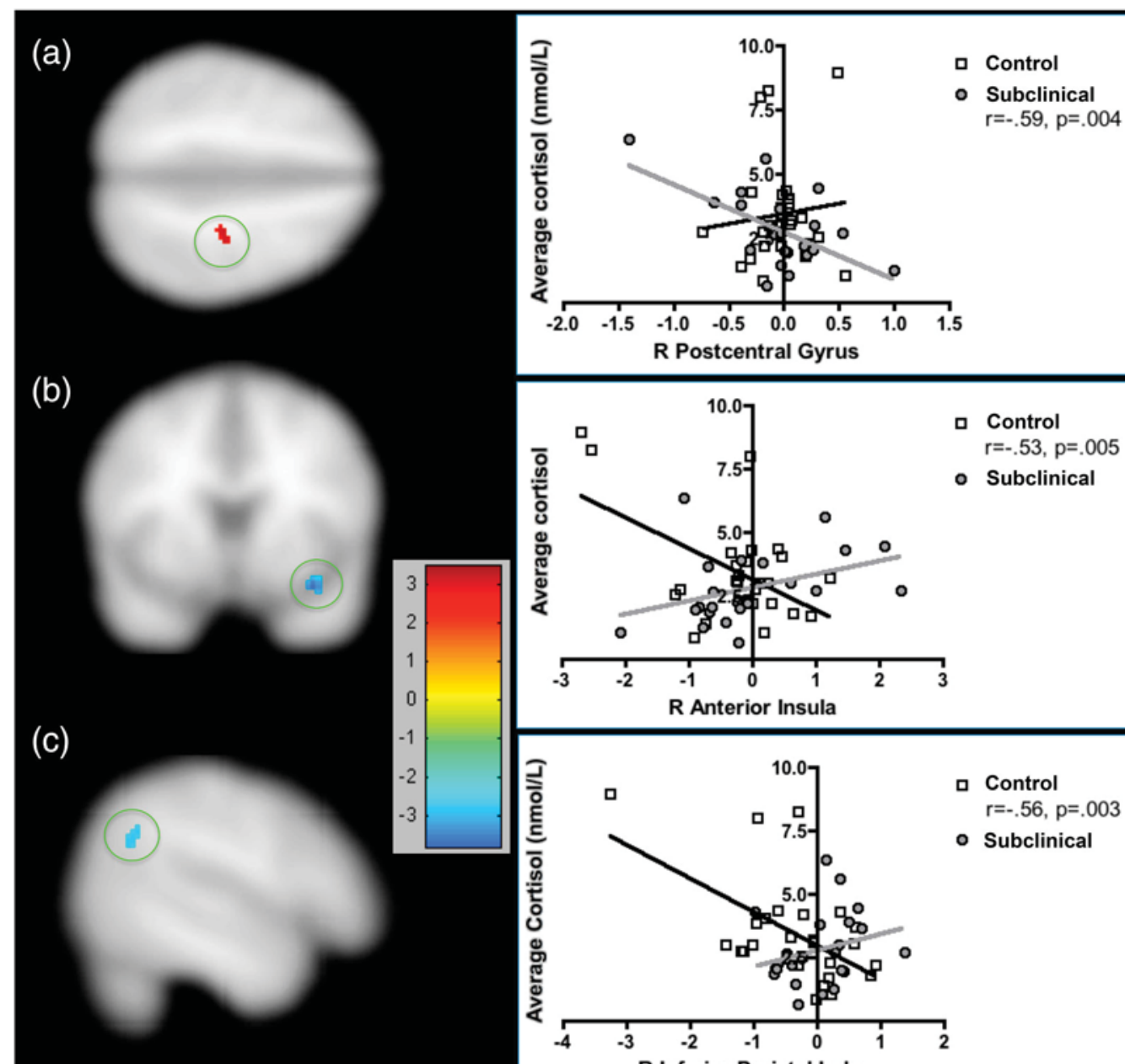

a Control $r=-.53, p=.005$

- Subclinical

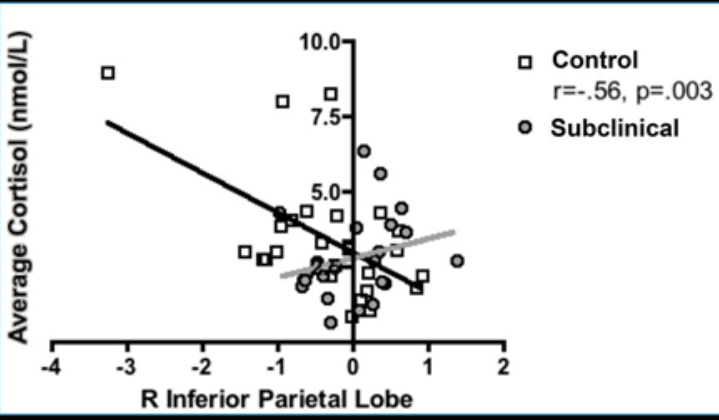

(d)

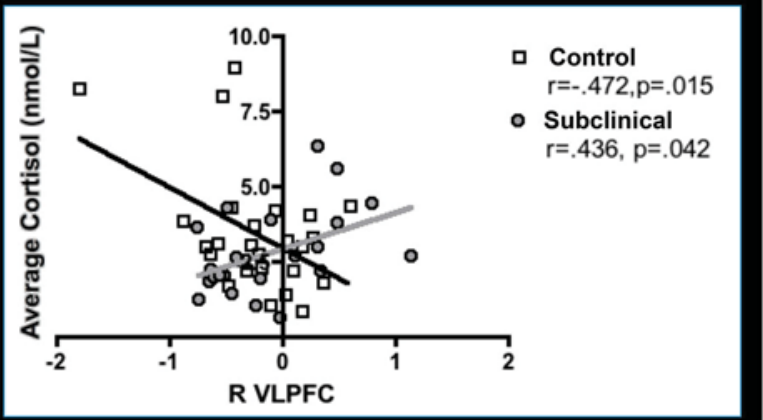

Figure 3. Group differences in gray matter in association between average cortisol levels during the task and neural activity in incongruent $>$ congruent contrast for happy cue + target trials. Multiple regression analyses revealed a significant Control $>$ Subclinical effect in the right postcentral gyrus (Figure 3(a)). Also, the analyses revealed a significant Subclinical $>$ Control effect in the right anterior insula (Figure 3(b)), right inferior parietal lobe (Figure 3(c)) and right VLPFC (Figure 3(d)). The graphs simply illustrate the decomposed effect; $r$-values are used to illustrate the direction of any significant correlation effect as contained within the primary analysis. All clusters are thresholded at intensity threshold of $p<.005$, extent threshold of 20 voxels. Areas in red connote the Control > Subclinical contrast, while those in blue, the Subclinical > Control contrast. VLPFC, ventrolateral prefrontal cortex. 
that the attentional bias is a mixture of automatic and controlled processing when stimuli are presented at longer than $500 \mathrm{~ms}$ in duration (Cisler \& Koster, 2010), as was the case in our study.

Furthermore, heightened levels of anxiety prior to the task could have played a facilitative role in this process. Indeed, two recent studies reveal a positive interactive role of anxiety with respect to coping in a non-clinical anxiety sample, and psychosocial functioning in subclinical depression. Specifically, a study on the effect of coping style on gaze duration in a non-clinical anxious sample found that participants who are sensitive to threatening situations but are able, when necessary, to employ cognitive coping strategies (be it at the level of vigilance or arousal) to deal with threats, show a longer gaze toward happy faces when faces are presented for greater than $500 \mathrm{~ms}$ (Klucken et al., 2010). Moreover, another study on participants with mixed anxiety/depression (MADD) subthreshold levels (Spijker, Batelaan, de Graaf, \& Cuijpers, 2010) showed that those with pure subthreshold depression tended to show more role limitations due to emotional problems, and greater impairment in social functioning compared to mixed group and the pure anxiety group. Therefore, although the effect of MADD on psychosocial functioning was found to be modest (particularly given that it seems to be a transient state) (Spijker et al., 2010), it would appear that the presence of some anxiety could be beneficial in the subclinically depressed sample. In the present sample, this effect was observed regarding attentional bias.

Findings related to neural correlates of attentional bias toward happy faces in the subclinical group also offer some preliminary support for our proposition. Namely, we observed a positive association between engagement of left inferior parietal lobule and attentional bias in the subclinical group. This region is part of top-down attentional control network (Corbetta, Patel, \& Shulman, 2008; Hopfinger, Buonocore, \& Mangun, 2000) and has previously been found to be involved in the voluntary orientation toward a target stimulus (Corbetta et al., 2000). Furthermore, studies have suggested that inferior parietal lobe may influence processing of target information in fusiform region (reviewed in (Hopfinger et al., 2000). Increased activity in fusiform region has been observed in response to targets appearing in valid (congruent) compared to invalid position (incongruent) (Corbetta et al., 2000), reflecting enhanced processing of target stimuli that are within the attentional focus (Hopfinger et al., 2000). Findings of negative association between engagement of fusiform in incongruent > congruent contrast and attentional bias toward happy faces in the current study are consistent with the literature.

Furthermore, we observed positive associations between activity in striatum and attentional bias toward happy faces in both the healthy group and the subclinical group, albeit in different regions: the caudate in the healthy group and putamen in the subclinical group. In the Triadic Model of motivated behavior, striatum represents the approach system and underlies motivation, reward processing, and incentive learning (Ernst \& Fudge, 2009). Along these lines, a study in healthy participants reported recruitment of caudate nucleus in response to incongruent > congruent contrast for happy faces, suggesting the capacity of approach-related cues to engage the caudate and its potential role in reward-attention association (Lindstrom et al., 2009). In the current study, we replicate these findings and extend them to the subclinically depressed sample. Notably, the association between striatal regions and attentional bias in both groups may also reflect their potential role in mediating an association between motivation to engage in the task and the degree of attentional bias detected. As we have not assessed participants' motivation levels to engage in the task, this assumption remains a hypothesis to be tested in future studies.

It should be noted that we did not observe any association between cortisol levels during the attentional bias task and attentional bias toward happy faces in the subclinical group. The lack of association could be explained by the fact that we presented happy and sad stimuli for $1000 \mathrm{~ms}$ (as this was done in previous studies to assess attentional bias in depression), and that we assessed cortisol levels during the attentional bias task rather than in response to a stress task. Nevertheless, we did observe group differences with respect to association between average cortisol levels during the attentional bias task and neural activity during happy attentional bias processing in key regions subserving attentional processing: the right anterior insula, right VLPFC, right inferior parietal lobule, 
as well as right postcentral gyrus. The right anterior insula and VLPFC are part of the ventral attentional network involved in stimulus-driven control of attention; the right inferior parietal lobule is part of the same network as well as the dorsal attentional network, involved in top-down control of attention (Corbetta et al., 2008). Typically, a balance between these networks is maintained such that one can both sustain attention to the goal-directed task (dorsal system), as well as respond to unexpected, but relevant stimuli (primarily ventral system). Under demanding condition, however, it has been proposed that the ventral attention network is preferentially deactivated, likely due to a top-down control restricting the network response to narrow range of task-relevant stimuli (Aston-Jones \& Cohen, 2005). The negative association between cortisol levels and the key regions within the ventral system in the control group specifically, would seem to suggest that in the context of heightened levels of cortisol, the ventral system in the control group would be adaptively downregulated to limit unnecessary processing of information. On the other hand, in the subclinical group, heightened levels of cortisol might contribute to continued or increased contribution of the ventral system to information processing, which in the long run may prove to be too taxing. This hypothesis needs to be investigated in future studies.

Overall, the findings of the current study are in line with a recent review which proposed that resilience and vulnerability to mood disorders need to be envisioned as dynamic processes that exist even in those populations that are typically viewed only as examples of vulnerability (Waugh \& Koster, 2014). Importantly, Waugh and Koster suggest that given that even the traditionally vulnerable populations show traces of resilience, intervention strategies that load on these resiliency states and traits would likely prove most efficient in preventing future depressive episodes (Waugh \& Koster, 2014). To this end, the current study adds to a growing literature that suggests that attentional bias training toward positive information as well as training in employing positive coping strategies might be a viable intervention (Browning et al., 2012; Dandeneau, Baldwin, Baccus, Sakellaropoulo, \& Pruessner, 2007; Taylor et al., 2011; Waters, Pittaway, Mogg, Bradley, \& Pine, 2013) for some individuals showing depressive tendencies at subclinical levels. In addition, we cautiously suggest that the study also provides clues for further investigation of possible neural targets that might aid in mechanistic treatment refinement as has been previously done with respect to cognitive processing in a healthy sample (Fregni et al., 2005) and clinical depression (Ferrucci et al., 2009; Shiozawa et al., 2014). Future studies need to investigate these possibilities further.

\section{Limitations}

It should be noted that this study is limited by small sample sizes and a small number of trials. Also, the healthy group did not show a bias for either the happy or sad faces. While some studies have shown that the healthy controls show attentional bias toward happy faces or avoid sad faces (Joormann et al., 2007), there are also those that have not found attentional bias in healthy participants (for example Gotlib et al., 2004). It is possible that within the healthy group, level of motivation to complete the task or variability in certain personality traits previously shown to modulate levels of attentional bias within a healthy sample (such as for example levels of self-esteem; (Jevtic, Dedovic, \& Pruessner, 2012)) may have masked our ability to detect attentional bias in the healthy. In addition, even though two weeks had passed from the time participants completed the face-rating task to the time they saw the faces again in the dot-probe task, it might be possible that the impact of the stimuli was decreased due to habituation. Furthermore, the study is based on highly efficient, university educated, young adult population and therefore, results of this study may not generalize to all subclinical depression populations. Thus, results of this study would need to be replicated in other populations at-risk for depression. Furthermore, future studies with bigger sample sizes that will specifically examine the mechanisms of key attentional processes (shifting attention, attention disengagement, etc.) that underlie behavioral measure of attentional bias in subclinically depressed are needed. Moreover, coping strategies in this population were assessed by using a retrospective measure. 
In conclusion, this is a first study to examine association of several psychological, endocrine, and neural factors with attentional bias in subclinically depressed sample. Notably, the main results, which should be considered preliminary in nature, suggest that some subclinically depressed participants show traces of resilience in the form of attentional bias toward positive information; these characteristics should be examined further for their potential to inform the development of new integrative intervention strategies.

\section{Disclosure statement}

No potential conflict of interest was reported by the authors.

\section{References}

Aston-Jones, G., \& Cohen, J. D. (2005). An integrative theory of locus coeruleus-norepinephrine function: Adaptive gain and optimal performance. Annuual Review of Neuroscience, 28, 403-450. doi:10.1146/annurev.neuro.28.061604.135709

Beck, A. T., \& Steer, R. A. (1987). Manual for the beck depression inventory. San Antonio, TX: The Psychological Corporation. Bradley, B. P., Mogg, K., Falla, S. J., \& Hamilton, L. R. (1998). Attentional bias for threatening facial expressions in anxiety: Manipulation of stimulus duration. Cognition and Emotion, 12(6), 737-753.

Bradley, B. P., Mogg, K., \& Lee, S. C. (1997). Attentional biases for negative information in induced and naturally occurring dysphoria. Behaviour Research and Therapy, 35(10), 911-927. doi:10.1016/S0005-7967(97)00053-3

Browning, M., Holmes, E. A., Charles, M., Cowen, P. J., \& Harmer, C. J. (2012). Using attentional bias modification as a cognitive vaccine against depression. Biological Psychiatry, 72(7), 572-579. doi:10.1016/j.biopsych.2012.04.014

Buodo, G., Mento, G., Sarlo, M., \& Palomba, D. (2015). Neural correlates of attention to emotional facial expressions in dysphoria. Cognition and Emotion, 29(4), 604-620. doi:10.1080/02699931.2014.926862

Cisler, J. M., \& Koster, E. H. W. (2010). Mechanisms of attentional biases towards threat in anxiety disorders: An integrative review. Clinical Psychology Review, 30(2), 203-216. doi:10.1016/j.cpr.2009.11.003

Corbetta, M., Kincade, J. M., Ollinger, J. M., McAvoy, M. P., \& Shulman, G. L. (2000). Voluntary orienting is dissociated from target detection in human posterior parietal cortex. Nature Neuroscience, 3(3), 292-297. doi:10.1038/73009

Corbetta, M., Patel, G., \& Shulman, G. L. (2008). The reorienting system of the human brain: From environment to theory of mind. Neuron, 58(3), 306-324. doi:10.1016/j.neuron.2008.04.017

Dandeneau, S. D., Baldwin, M. W., Baccus, J. R., Sakellaropoulo, M., \& Pruessner, J. C. (2007). Cutting stress off at the pass: Reducing vigilance and responsiveness to social threat by manipulating attention. Journal of Personality and Social Psychology, 93(4), 651-666.

Dedovic, K., Duchesne, A., Engert, V., Lue, S. D., Andrews, J., \& Efanov, S. I. (2014). Psychological, endocrine and neural responses to social evaluation in subclinical depression. Social Cognitive and Affective Neuroscience, 9(10), 16321644. doi:10.1093/scan/nst151

Dedovic, K., Renwick, R., Mahani, N. K., Engert, V., Lupien, S. J., \& Pruessner, J. C. (2005). The Montreal imaging stress task: Using functional imaging to investigate the effects of perceiving and processing psychosocial stress in the human brain. Journal of Psychiatry \& Neuroscience, 30(5), 319-325.

Dressendorfer, R. A., Kirschbaum, C., Rohde, W., Stahl, F., \& Strasburger, C. J. (1992). Synthesis of a cortisol-biotin conjugate and evaluation as a tracer in an immunoassay for salivary cortisol measurement. The Journal of Steroid Biochemistry and Molecular Biology, 43(7), 683-692.

Ernst, M., \& Fudge, J. L. (2009). A developmental neurobiological model of motivated behavior: Anatomy, connectivity and ontogeny of the triadic nodes. Neuroscience and Biobehavioral Reviews, 33(3), 367-382. doi:10.1016/j.neubiorev. 2008.10.009

Ferrucci, R., Bortolomasi, M., Vergari, M., Tadini, L., Salvoro, B., \& Giacopuzzi, M. (2009). Transcranial direct current stimulation in severe, drug-resistant major depression. Journal of Affective Disorders, 118(1-3), 215-219. doi:10.1016/j.jad. 2009.02.015

Foland-Ross, L. C., \& Gotlib, I. H. (2012). Cognitive and neural aspects of information processing in major depressive disorder: An integrative perspective. Frontiers in Psychology, 3, 1-17. doi:10.3389/fpsyg.2012.00489

Folkman, S., \& Lazarous, R. S. (1985). If it changes it must be a process: Study of emotion and coping during three stages of a college examination. Journal of Personality and Social Psychology, 48(1), 150-170. doi:10.1037/0022-3514.48.1.150

Fregni, F., Boggio, P. S., Nitsche, M., Bermpohl, F., Antal, A., \& Feredoes, E. (2005). Anodal transcranial direct current stimulation of prefrontal cortex enhances working memory. Experimental Brain Research, 166(1), 23-30. doi:10.1007/s00221005-2334-6

Gotlib, I. H., \& Joormann, J. (2010). Cognition and depression: Current status and future directions. Annual Review of Clinical Psychology, 6, 285-312. doi:10.1146/annurev.clinpsy.121208.131305 
Gotlib, I. H., Krasnoperova, E., Yue, D. N., \& Joormann, J. (2004). Attentional biases for negative interpersonal stimuli in clinical depression. Journal of Abnormal Psychology, 113(1), 127-135. doi:10.1037/0021-843X.113.1.127

Gotlib, I. H., McLachian, A. L., \& Katz, A. N. (1988). Biases in visual attention in depressed and nondepressed individuals. Cognition and Emotion, 2(3), 185-200. doi:10.1080/02699938808410923

Helzer, E. G., Connor-Smith, J. K., \& Reed, M. A. (2009). Traits, states, and attentional gates: Temperament and threat relevance as predictors of attentional bias to social threat. Anxiety, Stress, \& Coping, 22(1), 57-76. doi:10.1080/ 10615800802272244

Hopfinger, J. B., Buonocore, M. H., \& Mangun, G. R. (2000). The neural mechanisms of top-down attentional control. Nature Neuroscience, 3(3), 284-291. doi:10.1038/72999

Jenkinson, M., Bannister, P., Brady, M., \& Smith, S. (2002). Improved optimization for the robust and accurate linear registration and motion correction of brain images. Neuroimage, 17(2), 825-841. doi:10.1006/nimg.2002.1132

Jevtic, I., Dedovic, K., \& Pruessner, J. C. (2012). Examining the link between trait levels of self-esteem, state concentration levels and attentional bias towards happy and sad faces in healthy young adults. San Diego, CA: Society for Personality and Social Psychology.

Joormann, J., Talbot, L., \& Gotlib, I. H. (2007). Biased processing of emotional information in girls at risk for depression. Journal of Abnormal Psychology, 116(1), 135-143. doi:10.1037/0021-843X.116.1.135

Karsten, J., Hartman, C. A., Ormel, J., Nolen, W. A., \& Penninx, B. W. J. H. (2010). Subthreshold depression based on functional impairment better defined by symptom severity than by number of DSM-IV symptoms. Journal of Affective Disorders, 123(1-3), 230-237. doi:10.1016/j.jad.2009.10.013

Klucken, T., Brouwer, A. M., Chatziastros, A., Kagerer, S., Netter, P., \& Hennig, J. (2010). The impact of coping style on gaze duration. PLoS One, 5(11), e15395. doi:10.1371/journal.pone.0015395

Lewinsohn, P. M., Klein, D. N., Durbin, E. C., Seeley, J. R., \& Rohde, P. (2003). Family study of subthreshold depressive symptoms: Risk factor for MDD? Journal of Affective Disorders, 77(2), 149-157. doi:10.1016/S0165-0327(02)00106-4

Lieberman, M. D., \& Cunningham, W. A. (2009). Type I and Type II error concerns in fMRI research: Re-balancing the scale. Social Cognitive and Affective Neuroscience, 4(4), 423-428. doi:10.1093/scan/nsp052

Lindstrom, K. M., Guyer, A. E., Mogg, K., Bradley, B. P., Fox, N. A., \& Ernst, M. (2009). Normative data on development of neural and behavioral mechanisms underlying attention orienting toward social-emotional stimuli: An exploratory study. Brain Research, 1292, 61-70. doi:10.1016/j.brainres.2009.07.045

Luecken, L. J., Tartaro, J., \& Appelhans, B. (2004). Strategic coping responses and attentional biases. Cognitive Therapy and Research, 28(1), 23-37. doi:10.1023/B:COTR.0000016928.98893.fb

Lundqvist, D., Flykt, A., \& Ohman, A. (1998). Karolinska directed emotional faces-database of standardized facial images. Sweden: Department of Clinical Neuroscience, Karolinska Hospital, Stockholm.

MacLeod, C., Mathews, A., \& Tata, P. (1986). Attentional bias in emotional disorders. Journal of Abnormal Psychology, 95(1), 15-20. doi:10.1037/0021-843X.95.1.15

Mathews, A., \& MacLeod, C. (1985). Selective processing of threat cues in anxiety states. Behaviour Research and Therapy, 23(5), 563-569. doi:10.1016/0005-7967(85)90104-4

Mathews, A., \& MacLeod, C. (2005). Cognitive vulnerability to emotional disorders. Annual Review of Clinical Psychology, 1, 167-195. doi:10.1146/annurev.clinpsy.1.102803.143916

McHugh, K. R., Behar, E., Gutner, C. A., Geem, D., \& Otto, M. W. (2010). Cortisol, stress, and attentional bias toward threat. Anxiety, Stress, \& Coping, 23(5), 529-545. doi:10.1080/10615801003596969

McNair, D. M., Lorr, M., \& Droppleman, L. P. (1992). EdITS manual for the profile of mood states. San Diego, CA: Educational and Industrial Testing Service.

Mitterschiffthaler, M. T., Williams, S. C., Walsh, N. D., Cleare, A. J., Donaldson, C., \& Scott, J. (2008). Neural basis of the emotional Stroop interference effect in major depression. Psychological Medicine, 38(2), 247-256. doi:10.1017/ S0033291707001523

Mogg, K., Bradley, B. P., \& Williams, R. (1995). Attentional bias in anxiety and depression: The role of awareness. British Journal of Clinical Psychology, 34(1), 17-36. doi:10.1111/j.2044-8260.1995.tb01434.x

Mogg, K., Mathews, A., \& Weinman, J. (1989). Selective processing of threat cues in anxiety states: A replication. Behaviour Research and Therapy, 27(4), 317-323. doi:10.1016/0005-7967(89)90001-6

Peckham, A. D., McHugh, K. R., \& Otto, M. W. (2010). A meta-analysis of the magnitude of biased attention in depression. Depression and Anxiety, 27(12), 1135-1142. doi:10.1002/da.20755

Price, R. B., Siegle, G. J., Silk, J. S., Ladouceur, C. D., McFarland, A., \& Dahl, R. E. (2014). Looking under the hood of the dotprobe task: An fMRI study in anxious youth. Depression and Anxiety, 31(3), 178-187. doi:10.1002/da.22255

Reynolds, W. (1995). Hamilton depression inventory: A self-report version of the Hamilton depression rating scale. Odessa, FL: Psychological Assessment Resources.

Rivas-Vazquez, R. A., Saffa-Biller, D., Ruiz, I., Blais, M. A., \& Rivas-Vazquez, A. (2004). Current issues in anxiety and depression: Comorbid, mixed, and subthreshold disorders. Professional Psychology: Research and Practice, 35(1), 7483. doi:10.1037/0735-7028.35.1.74

Salemink, E., van den Hout, M. A., \& Kindt, M. (2007). Selective attention and threat: Quick orienting versus slow disengagement and two versions of the dot probe task. Behaviour Research and Therapy, 45(3), 607-615. doi:10.1016/j.brat. 2006.04.004 
Shane, M. S., \& Peterson, J. B. (2007). An evaluation of early and late stage attentional processing of positive and negative information in dysphoria. Cognitiion and Emotion, 21(4), 789-815. doi:10.1080/02699930600843197

Shankman, S. A., Lewinsohn, P. M., Klein, D. N., Small, J. W., Seeley, J. R., \& Altman, S. E. (2009). Subthreshold conditions as precursors for full syndrome disorders: A 15-year longitudinal study of multiple diagnostic classes. Journal of Child Psychology and Psychiatry, 50(12), 1485-1494. doi:10.1111/j.1469-7610.2009.02117.x

Sheppard, L. C., \& Teasdale, J. D. (2004). How does dysfunctional thinking decrease during recovery from major depression? Journal of Abnormal Psychology, 113(1), 64-71.

Shiozawa, P., Fregni, F., Benseñor, I. M., Lotufo, P. A., Berlim, M. T., \& Daskalakis, J. Z. (2014). Transcranial direct current stimulation for major depression: An updated systematic review and meta-analysis. The International Journal of Neuropsychopharmacology, 17(9), 1443-1452. doi:10.1017/S1461145714000418

Smith, S. M., Jenkinson, M., Woolrich, M. W., Beckmann, C. F., Behrens, T. E. J., \& Johansen-Berg, H. (2004). Advances in functional and structural MR image analysis and implementation as FSL. Neuroimage, 23(Suppl. 1), S208-S219. doi:10.1016/j.neuroimage.2004.07.051

Solomon, A., Haaga, D. A. F., \& Arnow, B. A. (2001). Is clinical depression distinct from subthreshold depressive symptoms? A review of the continuity issue in depression research. The Journal of Nervous and Mental Disease, 189(8), 498-506. doi:10.1097/00005053-200108000-00002

Spijker, J., Batelaan, N., de Graaf, R., \& Cuijpers, P. (2010). Who is MADD? Mixed anxiety depressive disorder in the general population. Journal of Affective Disorders, 121(1-2), 180-183. doi:10.1016/j.jad.2009.06.008

Svanborg, P., \& Asberg, M. (1994). A new self-rating scale for depression and anxiety states based on the Comprehensive Psychopathological Rating Scale. Acta Psychiatrica Scandinavica, 89(1), 21-28. doi:10.1111/j.1600-0447.1994.tb01480.x

Taylor, C. T., Bomyea, J., \& Amir, N. (2011). Malleability of attentional bias for positive emotional information and anxiety vulnerability. Emotion, 11(1), 127-138. doi:10.1037/a0021301

Tsumura, H., \& Shimada, H. (2012). Acutely elevated cortisol in response to stressor is associated with attentional bias toward depression-related stimuli but is not associated with attentional function. Applied Psychophysiology and Biofeedback, 37(1), 19-29. doi:10.1007/s10484-011-9172-z

Vuilleumier, P. (2005). How brains beware: Neural mechanisms of emotional attention. Trends in Cognitive Sciences, 9(12), 585-594. doi:10.1016/j.tics.2005.10.011

Waters, A. M., Pittaway, M., Mogg, K., Bradley, B. P., \& Pine, D. S. (2013). Attention training towards positive stimuli in clinically anxious children. Developmental Cognitive Neuroscience, 4, 77-84. doi:10.1016/j.dcn.2012.09.004

Waugh, C. E., \& Koster, E. H. W. (2014). A resilience framework for promoting stable remission from depression. Clinical Psychology Review. doi:10.1016/j.cpr.2014.05.004

Woolrich, M. W., Jbabdi, S., Patenaude, B., Chappell, M., Makni, S., \& Behrens, T. (2009). Bayesian analysis of neuroimaging data in FSL. Neuroimage, 45(1, Suppl. 1), S173-S186. doi:10.1016/j.neuroimage.2008.10.055 